\title{
Surface Flow Field Measurement Based on Optical Flow
}

\author{
ZHANG yu ${ }^{1, a *} \quad$ JIANG Qinghui ${ }^{2, b}$ \\ 1.Yangtze River Scientific Research Institute, Wuhan 430010, China \\ 2.School of Civil Engineering Wuhan University, Wuhan 430072,China
}

a. zhangyu_1999@126.com

b. 29372440@qq.com

\section{Key words: Surface Displacement, Particle Image Velocimetry, Optical Flow}

Abstract. Non-contact surface flow field measurementhas been widely applied in many engineering applications. This study developed a channel instantaneous flow field measurement system based on calculation of optical flow. Some key issues including spatiotemporal filtering and additive fusion, perspective correction and lens correction, and the mutual correlation matching are discussed in detail. The corresponding treatment scheme has been provided in the developed measurement system. Finally, the system test results are given.

\section{Introduction}

Non-contact surface displacement measurement is a nondestructive measurement technique and has many applications in many fields such as water dynamics, aerodynamics, civil engineering, machinery manufacturing, etc. Particle image velocimetry (Particle Image Velocimetry, Acronym PIV [1]) is a typical non-contact surface displacement measurement system. The basic principle is: CCD imaging is used to obtain the continuous multi-frame images of the measured object, and the displacement of its surface is analyzed through the image analysis technology. Finally, the instantaneous velocity field is achieved by video analysis. The PIV system consists of computer, CCD camera, synchronous controller, and laser illumination system. According to the different practical purposes, CCD camera can be a consumer digital camera, high-speed industrial camera, video camera, etc. In the three-dimensional flow field test, it also needs to use several cameras focusing from different angles. Usually, the synchronization controller and laser lighting system can be omitted, with natural light and fixed lights instead. Measured object such as flow must have characteristics that can be detected from image. The displacement field is the distance between the same feature points which get from different frames. If no obvious feature points exist, the tracer particles should be create artificially as feature points. For example, In order to meet the requirements of flow measurement, floating particles should be uniformly distributed in the flow field. But limited by measurement density and operation complexity, the tracer particles method cannot get satisfactory results. In this paper, a channel instantaneous flow field measurement system is presented, which is based on calculation of optical flow. Some key issues in the developed system are discussed and the corresponding treatment scheme has been provided. Finally, the system test results are given.

\section{Introduction of the PIV system}

Large scale physical model experiment is an important technical means to solve the complicated problem of flow, but flow meter is far from meeting the need of measuring precision and density. As mentioned above, the PIV technology based on the tracer particles can be used to realize the measurement of 2D instantaneous flow field. In the physical model experiments, a large number of tracer particles are need be placed in the fluid. The tracer particles move in the fluid and indicate the movement of the flow field. But the number of the tracer particles increases sharply with the increase of the size of the physical model. A CCD camera is placed on the top of the fluid to capture the motion of flow. Based on the principle of correlation, the instantaneous particle displacement can be obtained by matching points in two consecutive frames. In our experiment, the random texture is generated by 
sprinkling the particles and dust on the surface of flow. These random textures are used to calculate the optical flow. Considering the smoothness of the surface displacement of the flow field, the flow velocity of the flow field can be measured by the optical flow of PIV system. Functions of the PIV system, such as file management, video display, video processing, vector processing and post-processing, is shown in Figure 1. This is an offline system. After the video data acquisition, the video data are selected to calculate and analyze. The interface of system is shown in figure 2 .

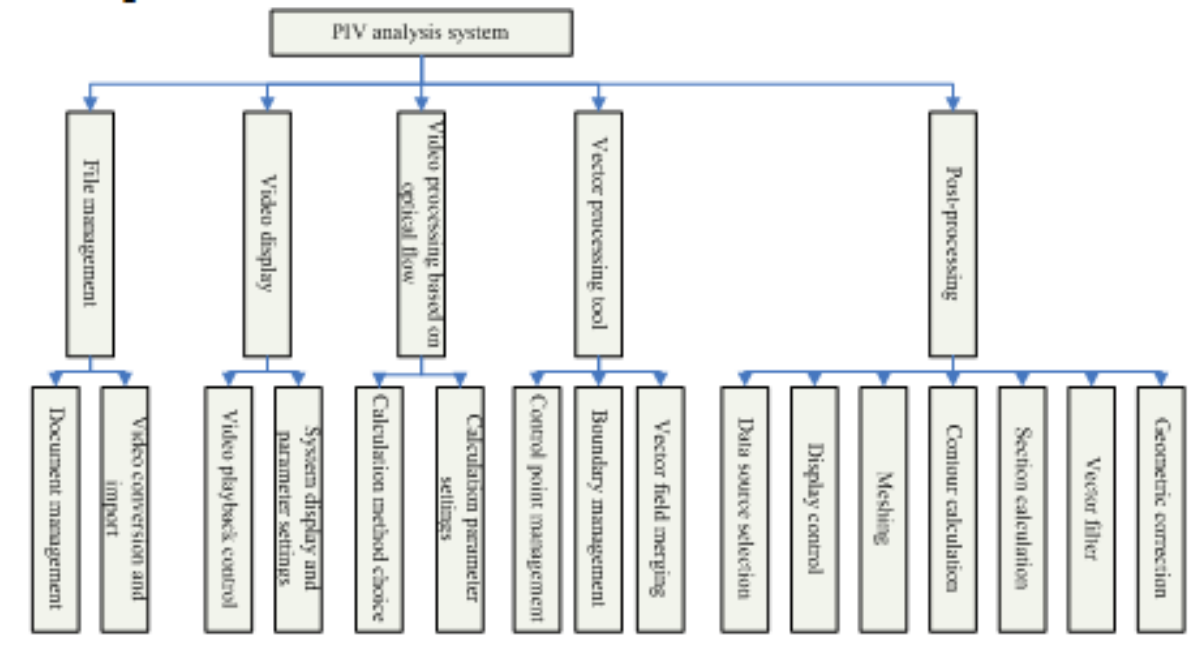

Fig.1 Functions of PIV

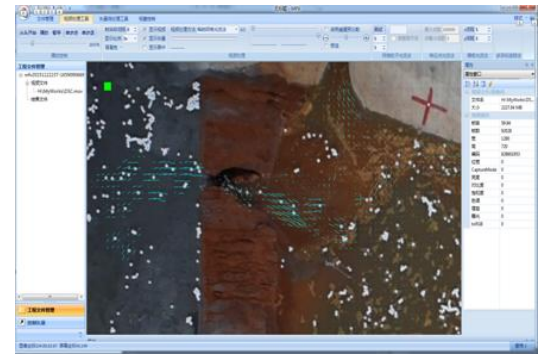

(a) Main system

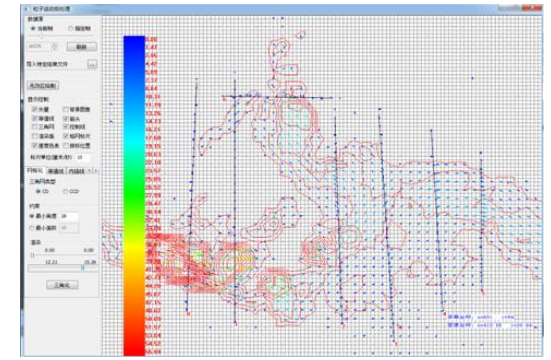

(b) Post-processing system

Fig.2 Interface of PIV System

\section{Key Issues}

\section{Spatiotemporal filtering and additive fusion}

In the flow measurement based on PIV, some errors occurred in the velocity measurement. The cause of the error can be divided into two categories: 1) Errors caused by the video content such as illumination changes of the scene, reflection of the wave and the CCD noise; 2) Optical flow cannot detect large changes of two consecutive frames. These errors can be corrected by spatiotemporal filtering. In the spatial domain, based on the principle of Gauss smoothing, the data of each velocity measurement results are processed in intensity and direction of vectors. First, the isolated noise points are detected and removed, and then a Gauss template is used to smooth the results. It can be considered that fluid status maintain a constant in a short time. Due to the invariance of flow field in a short time of the video sequence, an effective method is to adopt smooth filtering for the same point on all frames within this period, which can eliminate the noise generated by the mutation in time. The result of spatiotemporal filtering is shown in figure 3. The multi-resolution method can be used to fuse the results of optical flow, which can be used to break the limitation of optical flow algorithm. The video frame is sampled with different frequency that the high frequency frame is used to detect the high speed flow while the low frequency frame is used for slow part in the video. By additive 
fusion of several results different frequencies, it can be used to measure the large variation of the flow field. The overall flow chart is shown in figure 4.
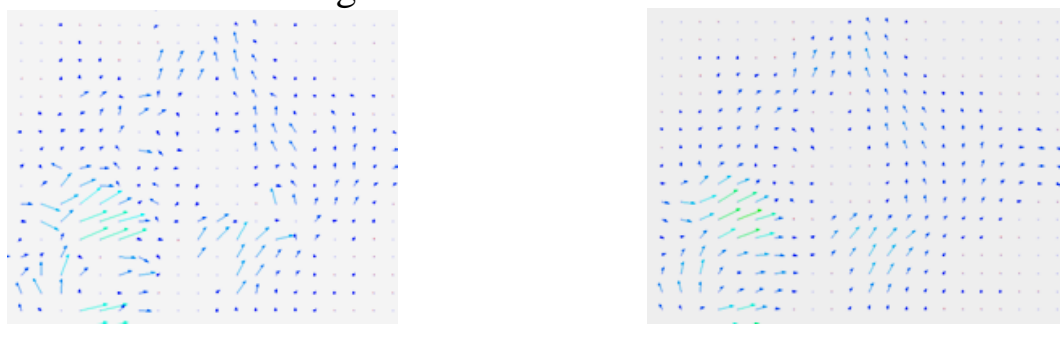

Fig. 3 Result of spatiotemporal filtering

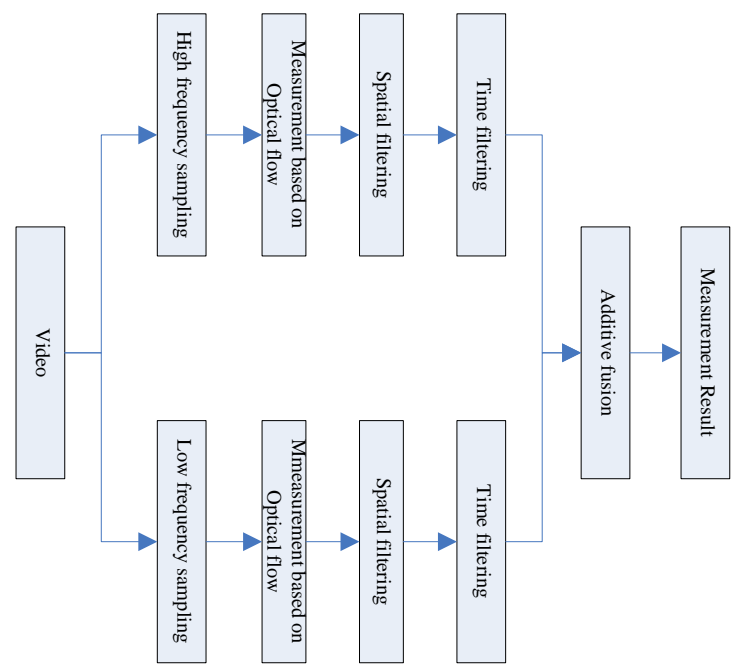

Fig.4 Flow chart of spatiotemporal filtering and additive fusion

\section{Calculation of optical flow}

In the PIV system, the mutual correlation matching means that the same point strength between two consecutive frames does not change with time. The fluid is generally a smooth surface. The velocity flow field adjacent positions do not change, Thereby forming two basic optical flow constraint equations:

$$
\begin{aligned}
& \frac{\partial I}{\partial x} u+\frac{\partial I}{\partial y} v+\frac{\partial I}{\partial t}=0 \\
& \left(u-u_{c}\right)^{2}+\left(v-v_{c}\right)^{2}=0
\end{aligned}
$$

Where $I$ is the current point image intensity, $(u, v)$ is the current displacement velocity vectors, $\left(u_{c}, v_{c}\right)$ is the mean of neighborhood of the $(\mathrm{u}, \mathrm{v})$. Because of the existence of different smoothing constraints, optical flow computation has many forms. Optical flow calculation must also maintain tiny displacement of two adjacent frames, which led to failure of detecting large scale displacement. This is why a multi-scale frame frequency is applied, as described above. Lucas-Kanade method [2] is a widely used and is a sparse optical flow method based on feature points. Due to the structure of multi-scale pyramid, it expanded the scope of the displacement which can be calculated. Figure 5 (a) shows the PIV displacement vector based on the Lucas-Kanade optical flow algorithm. The other is dense optical flow, which requires the calculation of the displacement vector of each pixel, as introduced in [3]. By using the quadratic polynomials to approximate local neighborhood of the image, the displacement field of the adjacent frames is obtained by polynomial coefficients. Figure 5 (b) shows the results of the dense optical flow with an interval sampling of the dense flow in order to facilitate the visualization of dense optical flow in the graph. 


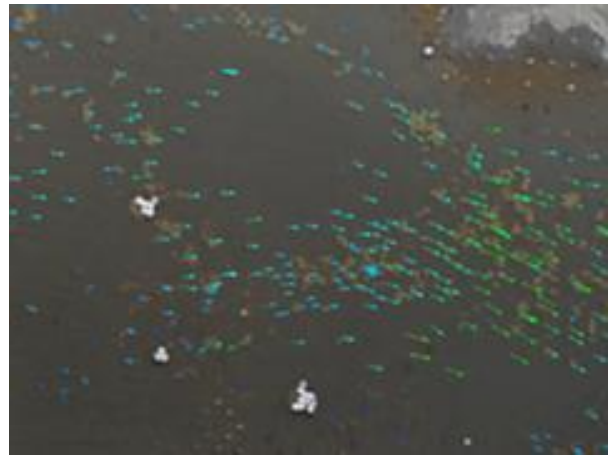

(a)

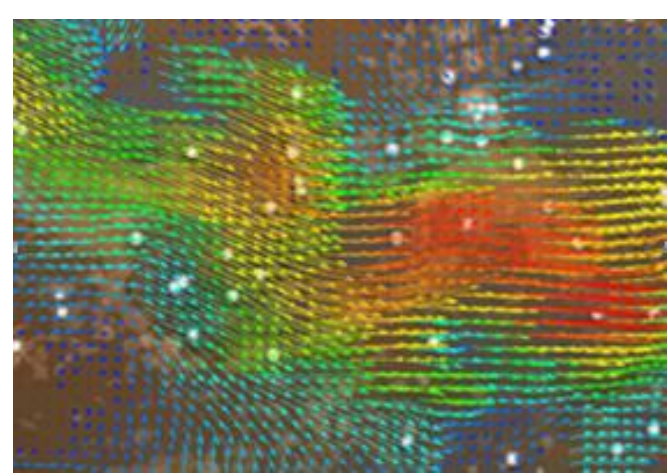

(b)

Fig.5 Vector field obtained based on optical flow

Optical flow itself has certain smoothness, which is suitable for the detection of flow field. When the flow field is around the tiny floating dirt, the details of the instantaneous movement can be obtained by the calculation of dense optical flow field, which makes the ability of such algorithms to measure natural river, lake, ocean currents and ice, etc.

\section{Perspective correction and lens correction}

Perspective correction

In actual test scenarios, the CCD camera is generally not perpendicular to the surface. Therefore, the image is the result of the perspective projection and has a perspective distortion. If four or more points on the same plane in test scenarios are known, the perspective distortion can be eliminated by projective transformation. In the image, the four image points are identified, and the corresponding coordinates in test scenarios is $X^{\prime}$, then the corresponding:

$$
X^{\prime}=H X
$$

Where $\mathrm{H}$ is a $3 \times 3$ matrix and has a total of 8 degrees of freedom with removing a proportion factor. A set of corresponding points can provide two equations, the four set of points can provide eight equations, sufficient to solve all the independent elements of $\mathrm{H}$. When the instantaneous flow field with large area is measured by the PIV system, it is very difficult to meet the requirement of the test. Therefore, camera can only be placed in a relatively high position with tilt shoot, which led to obvious perspective image distortion. By giving the corresponding practical control points on the same plane of the physical model, we can realize the correction of the perspective distortion, as shown in Figure 6.
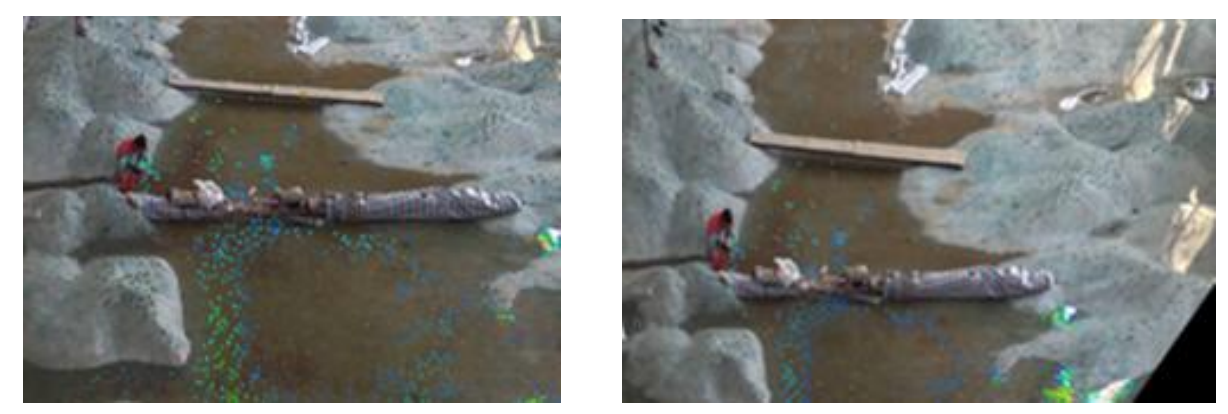

Fig.6 Perspective correction

Lens correction

CCD imaging based surface measurement requires the use of optical lenses, thus, optical distortion cannot be avoided. The main distortion of the lens is radial distortion, which is expressed as follows:

$$
\begin{aligned}
& x^{\prime}=x_{c}+L(r)\left(x-x_{c}\right) \\
& y^{\prime}=y_{c}+L(r)\left(y-y_{c}\right)
\end{aligned}
$$


The coordinate of image with radial distortion is $(x, y),\left(x_{c}, y_{c}\right)$ is the image center, and the coordinates $\left(x^{\prime}, y^{\prime}\right)$ is ideal position, as shown in Figure 7.

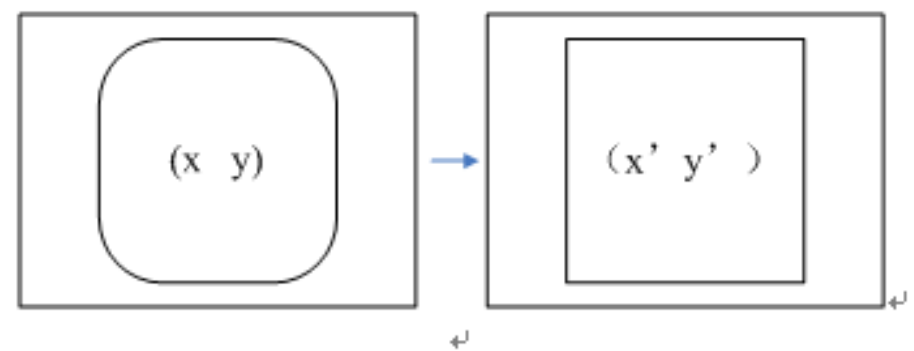

Fig.7 Lens Distortion Correction

$L(r)$ defines a distortion factor, $\mathrm{r}$ is the radius. $L(r)$ is defined as:

$$
L(r)=1+k_{1} r+k_{2} r^{2}+\cdots
$$

The camera parameters are defined by $\left(k_{1}, k_{2}, x_{c}, y_{c}\right)$. After calibration of the lens, the image can be sampled by (3) and the distortion caused by lens is removed.

Visualization of vector field

Vector field is used to express the displacement of corresponding points in the image sequence. The vector is visualized by a straight line and an arrow, which represents the vector strength and direction respectively, as shown in Figure 8 (a). The vector strength is also rendered by color. Strength here is a scalar, which can be defined as various forms such as vector strength, $\mathrm{x}$ direction displacement, y direction displacement, and so on. A mesh is generated by all vectors with different position. Every node of the mesh is rendered by different color according to its strength. Figure 8 (b-c) is the result of the triangulation and surface rendering. The contour lines can be generated by the mesh of triangulation. Because the flow surface is based on the structure of the triangular mesh, any point in the grid can be obtained by the interpolation of the triangle. Then, flow rate at any position of artificial input can be obtained by mesh interpolation, as shown in Figure 8 (d).

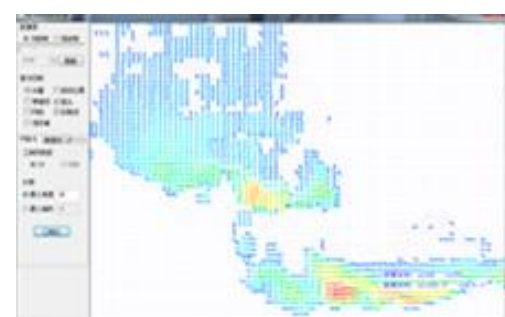

(a)

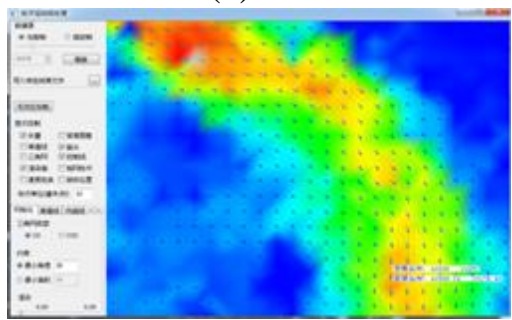

(c)

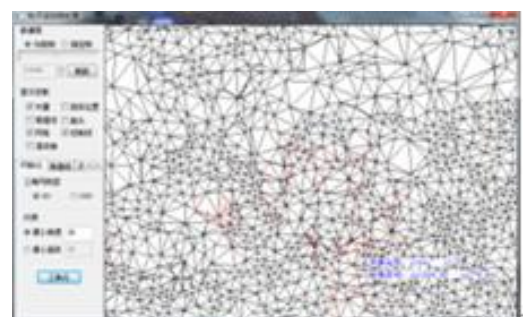

(b)

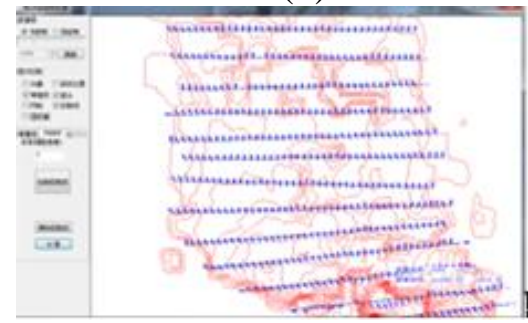

(d)

Fig.8 Visualization of vector field 


\section{Experiment and discussion}

The PIV system is used for studying the physical simulation of dam break. The purpose is to obtain the extent and influence of the flood. Physical test is located in indoor. In order to obtain a sufficient number of vectors, without affecting the test results, the mixture of particles and dust is added as tracer. The camera is placed in the top of the test hall. Control points are set up at different positions of the surface. By comparing with the flow velocity meter, the PIV analysis system can accurately capture the dense velocity through consecutive frames. However, as indicated by the literature [4], dam break occurred in all of a sudden outburst, the flow rate and the water level change dramatically in a very short period. In the dam break test, its maximum flow velocity can reach $1.5 \mathrm{~m} / \mathrm{s}$, but most of the flow field in the downstream is stable, and the relative velocity is only a few centimeters per second. Therefore, frame sampling with fixed frequency is easy to lead to the analysis error. As mention in the section 3.1, an effective solution is to use the time multi-resolution method which assuming that the flow field maintain a constant flow in a relatively short time. In the PIV system, different frame frequency is used to analyze the flow field with different flow speed. Final result is generated by additive select fusion. Figure 9 (a) shows a video image analysis results of that the low frequency sampling. Figure 9 (b) is an analysis result of high frequency sampling and the final fusion as shown in Figure 9 (C). By fusing the results of different frequency in a short time, the dense of vector field is increased and the analysis of flow rate with large change is possible.

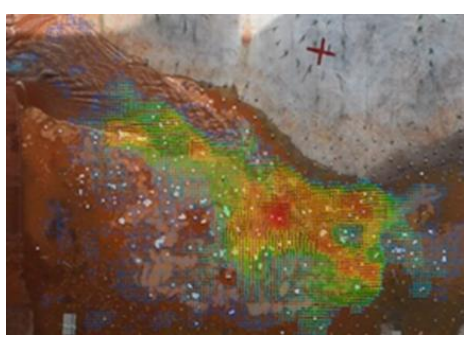

(a)

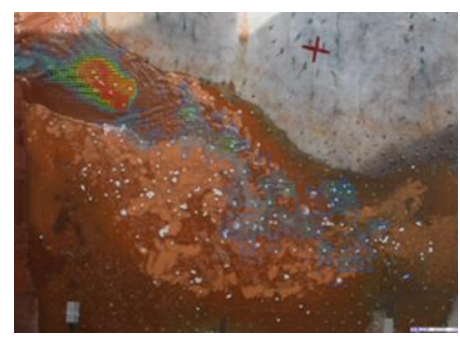

(b)

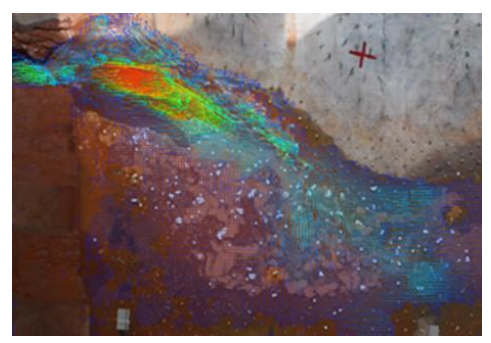

(c)

Fig.9 Additive fusion of vector field

\section{Conclusions}

Measuring based on image is belonging to the research field of vision measurement [5], which has a wide uses in many engineering applications. This study developed a channel instantaneous flow field measurement system based on the calculation of optical flow. Some key issues in the developed system are discussed and the corresponding treatment scheme has been provided. As a post-processing, a spatial and temporal method is applied. This makes it possible to analyze the large variation of the flow field. Test results proved that the PIV system mentioned above is effective.

\section{References}

[1]Ding Lihua, Zhang Xiaoguang, Zhao Liang, Zhang Haiming, Application of PIV Digital Image Testing Technology in TunDish Water Model Experiment[C], 2011 CSM Annual Meeting Proceedings ,2011.

[2] Lucas, B., and Kanade, T. An Iterative Image Registration Technique with an Application to Stereo Vision, Proc. of 7th International Joint Conference on Artificial Intelligence (IJCAI), pp. 674-679.

[3] Gunnar Farneback, Two-frame motion estimation based on polynomial expansion, Lecture Notes in Computer Science, 2003, (2749), 363-370. 
[4]WANG Li-hui, HU Si-yi Study on dam failure-realted problems [J] Advances in Science and Technology of Water Resources. 2007,01, Vol.27 No.1.

[5]ZHANG Guang-jun, Vision Measurement [M], Science Press. 2008 ISBN: 9787030209238 